\title{
Welche Folgen hat der Nikotinkonsum weltweit?
}

\author{
Während in den USA die Zahl der aktiven Raucher massiv \\ zurückgeht, betrifft der Nikotinabusus in Ländern wie China, \\ Russland und der Ukraine immer noch fast die Hälfte der \\ Bevölkerung. Erstmalig liegt mit der Studie von G. A. Giovino \\ et al. jetzt eine empirische Erhebung über das weltweite \\ Ausmaß des Nikotinabusus und seiner Begleitumstände vor. \\ Lancet 2012; 380: 668-679
}

Die Datengrundlage des Global Adult Tobacco Surveys (GATS) bilden repräsentative Interviews in 14 Entwicklungs- und Schwellenländern. Zusätzlich standen Daten aus Großbritannien und den USA zur Verfügung. Personen über 15 Jahre wurden über den Umfang, den Beginn und den aktuellen Status ihres Nikotinabusus befragt. Zusätzlich ermittelten die Autoren epidemiologische Basisdaten (z. B. Alter und Geschlecht). Somit steht erstmalig eine vergleichende, weltweite Erhebung zum Umfang des Nikotinmissbrauchs zur Verfügung. Anhand dieser Daten lassen sich auch Interventionsstrategien konzipieren.

In der Altersgruppe über 15 Jahre (3 Mrd. Menschen) rauchen weltweit etwa 852 Mio. Menschen. Bei der Analyse fanden die Autoren starke regional-kulturelle Unterschiede: So Rauchen in China zwar 53\% aller Männer, aber nur 2,4\% der Frauen. In den USA dagegen liegt das Verhältnis bei 24\% männlichen zu 16\% weiblichen Rauchern. In Schwellenländern wird tendenziell immer früher mit dem Rauchen begonnen. Auch hier ist China exemplarisch, da sich das Einstiegsalter von 24 auf mittlerweile 20 Jahre vermindert hat. In dieser Hinsicht nähert es sich der Situation in Großbritannien und den USA an. Hier be- ginnt die Raucherkarriere im Mittel über die Zeit konstant mit 18 Jahren. Nikotinabstinenz ist in den Schwellenländern eine Rarität. Nur 13\% aller Chinesen, 12\% aller Inder und 19\% aller Russen haben mit dem Rauchen aufgehört. Im Gegensatz dazu haben $57 \%$ aller Briten und $49 \%$ aller USAmerikaner den Nikotinkonsum aufgegeben. Auffallend ist zudem, dass auch in den Schwellenländern fast nur kommerzielle Zigaretten genutzt werden. Eine Ausnahme hiervon ist der dominierende Gebrauch von Kautabak in Indien.

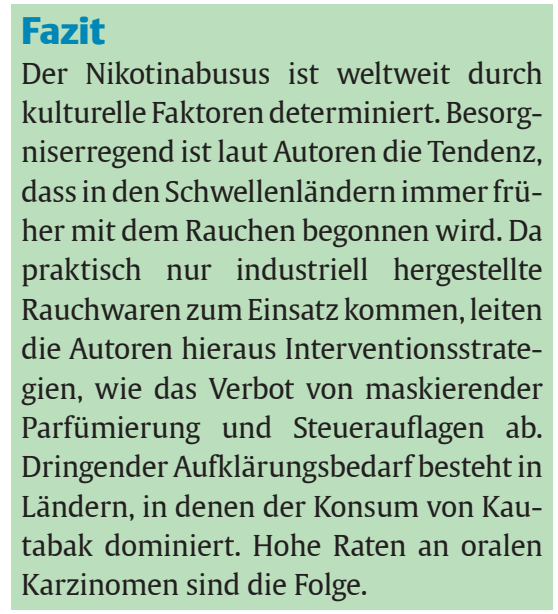

\section{Dr. Horst Gross, Berlin}

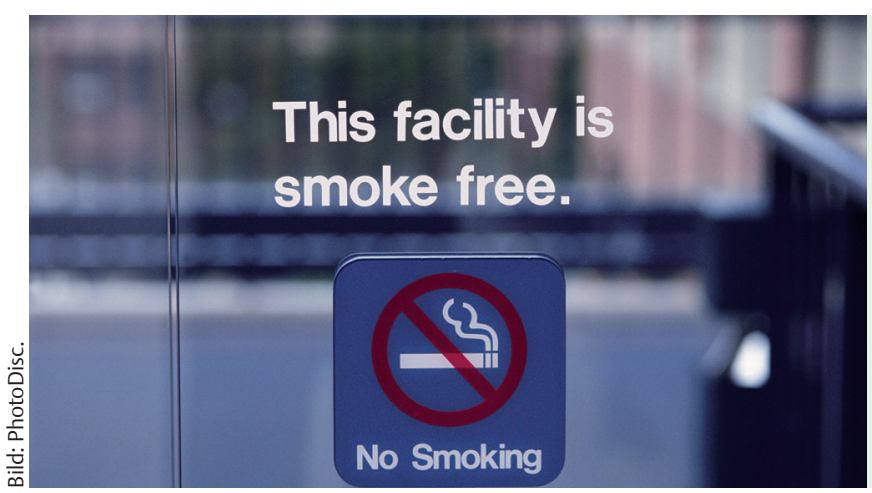

In China raucht jeder zweite Mann. „No smoking" heißt es dort nur für Frauen: Laut Studie greifen $2,4 \%$ der Chinesinnen zur Zigarette.
Allergologen haben nun belegt, dass Kinder mit einem niedrigen Geburtsgewicht innerhalb der ersten 6 Lebensjahre nicht unbedingt an Asthma erkranken. Die Medizin ging lange von diesem Zusammenhang aus. „Asthma ist die häufigste chronische Erkrankung im Kindesalter und ein häufiger Grund für Fehlzeiten in der Schule. Die Ursache für die Erkrankung ist immer noch unbekannt - dass es mit einem niedrigen Geburtsgewicht zusammenhängt, können wir ausschließen", sagt Studienleiter Hyeon Yang von der Mayo Clinic in Rochester, Minnesota.

\section{Fehlerhafte Statistik \\ $\nabla$}

Allein in den USA sind mehr als 25 Mio. Menschen von Asthma betroffen. Yang und sein Team untersuchten 3740 Menschen mit normalem Geburtsgewicht und 193 Personen mit niedrigem Geburtsgewicht. Die Probanden wurden im Zeitraum von 1976-1979 geboren. 6,7\% der Untergewichtigen entwickelten Asthma, bei den Kindern mit Normalgewicht waren es 5,4\%. Der Unterschied ist jedoch zu klein, um zu behaupten, dass ein niedriges Geburtsgewicht für die Entwicklung eines Asthmas relevant ist. „Die Zeitspanne der Geburten liegt ziemlich lange zurück. Wurde der Gesundheitszustand der Studienteilnehmer durch Befragungen eruiert, kann dies zu Fehlangaben führen“, kritisiert Sereina Maibach vom aha! Allergiezentrum in der Schweiz.

\section{Normalgewicht von Vorteil \\ $\nabla$}

„Auf Basis unserer Recherchen ist davon auszugehen, dass das Asthmarisiko im Zusammenhang mit dem Geburtsgewicht in einer U-förmigen Kurve verläuft - das heißt, dass bei tiefem und hohem Geburtsgewicht ein größeres Risiko für Asthma besteht", so Maibach. Die Experten schließen daraus, dass ein normales Geburtsgewicht dazu beitragen kann, präventiv das Risiko für Asthma so klein wie möglich zu halten.

Nach einer Mitteilung der pressetext Nachrichtenagentur $\mathrm{GmbH}$ 